\title{
Highly optically responsive divalent benzimidazolium-based axles with pseudorotaxane potential
}

Received 00th January 20xx, Accepted 00th January 20xx

DOI: $10.1039 / \times 0 \times x 00000 x$

\author{
S. Maryamdokht Taimoory*‡a,b, Xiao Yu ${ }^{\ddagger a}$, John F. Trant*a
}

\begin{abstract}
Mono and (bis)benzimidazoliums were evaluated both experimentally and computationally for their potential as building blocks of pseudopolyrotaxane axles. Their aggregation and optical behavior, along with their potential to form a [2]pseudorotaxane with dibenzyl-24-crown-8, was studied through the synergistic application of 1D/2D and diffusion ordered NMR spectroscopy, ultraviolet-visible \& fluorescence spectroscopy, and time-dependent density functional theory. Their unique optical behaviour was measured and modeled as a function of protonation state, solvent, and concentration. The axles show strong solvochromaticism and a very pronounced concentration-dependent optical profile. This axle with multiple recognition sites, has the potential to form pseudorotaxanes with tunable optical behavior.
\end{abstract}

\section{Introduction}

The tunability of heterocyclic chromophores continuously advances as new molecular scaffolds are added to the synthetic toolbox, which in turn drives the development of materials and devices with either improved performance or unprecedented function. ${ }^{[1]}$ Benzimidazole is a privileged motif, especially for solution state applications, ${ }^{[3]}$ exemplified by the biocompatible bis- benzimidazole fluorescent probe Hoechst 33258 (Figure 1), and fluorescent drug Albendazole. ${ }^{[4]}$

Benzimidazoles' properties, such as their electron accepting and $\pi$-bridging capacity, $\mathrm{pH}$ sensitivity/switching, ${ }^{[5]}$ ion chelating ability, ${ }^{[6]}$ and incorporation into biomolecules has made them attractive targets for a wide range of applications including optoelectronics, ${ }^{[7]}$ photovoltaics, ${ }^{[8]}$ solvatochromic probes, ${ }^{[9]}$ photocatalysts ${ }^{[10]}$, corrosion inhibitors ${ }^{[11]}$, and molecular recognition ${ }^{[12]}$.

It is in this last application that the multifunctional nature of the benzimidazoles truly comes into its own as their basicity and structural rigidity makes them potential guests for crown ether macrocycles. ${ }^{[13]}$ Bis-benzimidazoliums with two fluorophores in close proximity, but not in conjugation, offer significant opportunities for the design of new materials.[14] To our knowledge the aggregation, photochemical, and chemical behavior of bis-, tris-, and oligomeric-benzimidazole units with largely degenerate recognition sites has not been explored.

As a part of our interest in tunable supramolecular recognition systems, we have been aiming to prepare pseudorotaxanes incorporating novel bis(methylene-bridged-2arylbenzimidazoles) (bMeBABs) to exploit their exceptional binding affinity for crown ether macrocycles; [13a, 15], [16] benzimidazoles themselves are only moderately effective as crown ether templates, but substituted systems have well established affinities orders of magnitude higher with Kas on the order of $10^{5} \mathrm{M}^{-1}{ }^{[15]}$ However, we noted that our simple incorporation into optical chemical sensors for both solid, ${ }^{[2]}$ and

monomeric model systems, where the benzimidazoliums are connected through a methylene spacer, showed curious optical behavior (Figure 1). This work summarizes our exploration of these seemingly simple bMeBABs, including their optical, aggregative, and polymerization behavior and a preliminary investigation of their potential as axles for [2]pseudorotaxanes. ${ }^{[13 a-c, 14,17]}$ bMeBAB properties change as a function of ionization, concentration and solvent. Timedependent DFT (TD-DFT) calculations support the experimental results and demonstrate that the complex molecular orbitals on these systems lead to the observed properties and possible use as colour changing sensors. ${ }^{[18]}$

- Examples of benzimidazoles as dyes and drugs

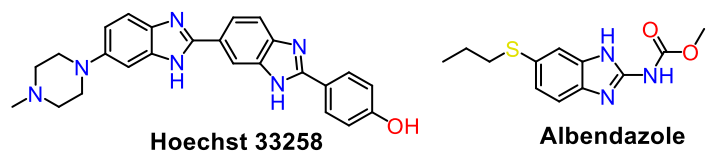

- This work:

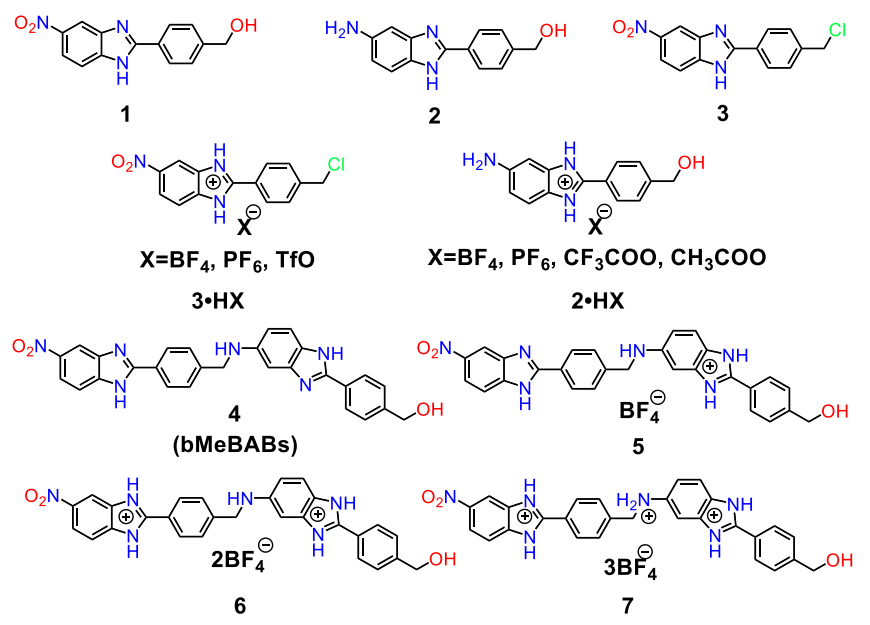

Figure 1. Some examples for previously reported benzimidazole derivatives and the novel bMeBABs and their precursors explored in this work.

\section{Results and Discussion}

ON N9B 3P4 Windsor, Canada; taimoory@uwindsor.ca j.trant@uwindsor.ca

${ }^{b}$. Department of Chemistry, University of Michigan, 930 N. University Ave., 2006B, Ann

Arbor, MI 48109.

* These authors contributed equally to this work and are listed in this order for alphabetical reasons alone, the names can be provided in any order for any professional purpose.

${ }^{+}$Electronic Supplementary Information (ESI) available: Experimental details; synthetic procedure, computational details, and characterization data. See DOI: XXXXXXXX
Photophysical behaviour varies greatly as a function of protonation state and charge density Benzimidazole precursor 1 was synthesized according to published procedures (See 


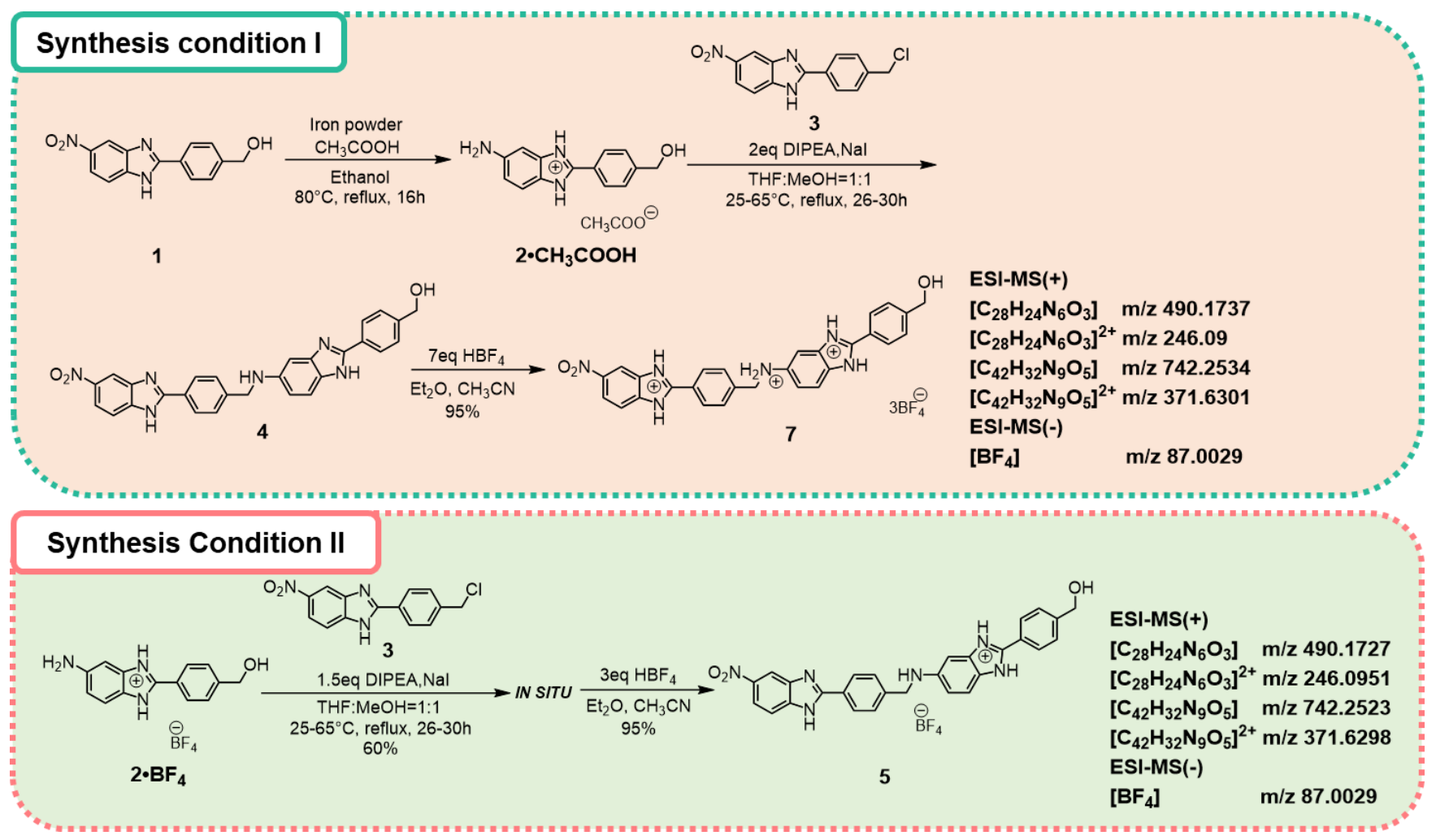

Scheme 1. Synthetic strategies used to access triprotonated $\mathbf{7}$, and mono-protonated $\mathbf{5}$, and neutral $\mathbf{4}$ from precedented 1.
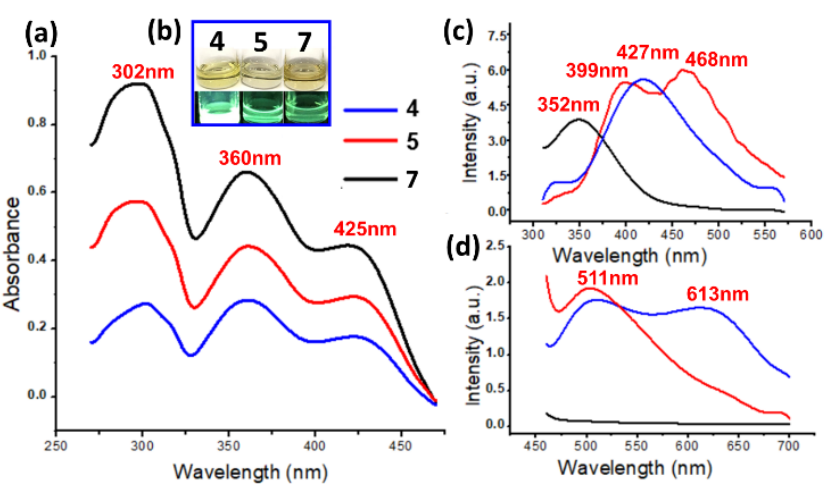

Figure 2. (a) The UV-Vis spectra of 4,5 and $\mathbf{7}(0.03 \mathrm{mM})$ in dry $\mathrm{DMSO}\left(\mathrm{d}_{6}\right)$; (b) pictures of 4,5 and $\mathbf{7}(0.03 \mathrm{mM})$ in pure dry DMSO $\left(d_{6}\right)$ under both ambient and $\lambda=365 \mathrm{~nm}$ light; The fluorescence spectra of $\mathbf{4}, \mathbf{5}$ and $\mathbf{7}(0.03 \mathrm{mM})$ in dry DMSO $\left(\mathrm{d}_{6}\right)$ when excited at either 300 (c) or $450 \mathrm{~nm}$ (d) excitation.

Supporting Information). [19] To access the bis-, tris-, oligomeric, and polymeric homologs, $\mathbf{1}$ was reduced to $\mathbf{2} \cdot \mathbf{C H}_{3} \mathbf{C O O H}$ (Scheme 1) and coupled with chloride 3 to provide bMeBAB 4 which features readily ionizable chromophores consisting of two phenyl-benzimidazoles separated by a methylene group.

We hypothesized that by tuning the amount of $\mathrm{HBF}_{4}$ added, we could control the degree of protonation of the system (Scheme 1). Consequently, neutral 4 was treated with $\mathrm{HBF}_{4}$ in acetonitrile at $25{ }^{\circ} \mathrm{C}$ using two different conditions and conversion to the benzimidazolium salts was monitored by ${ }^{1} \mathrm{H}$ NMR spectroscopy, confirmed using positive and negative mode ESI-MS, UV-Vis, and correlated with DFT calculations. The assigned fully tri-protonated $\mathbf{7}$ is accessed through addition of excess $\mathrm{HBF}_{4}$ ( 7 equiv.) to 4 in acetonitrile followed by precipitation with diethyl ether until no more precipitate crashed out of the solution. Using smaller amounts of $\mathrm{HBF}_{4}$ generated a mixture of 5, $\mathbf{6}$ and $\mathbf{7}$ which were challenging to characterize. Consequently, a different approach was required to provide better control. Under condition II, an already protonated monomer, $\mathbf{2} \cdot \mathbf{H B F}$, was coupled with $\mathbf{3}$ in the presence of 1.5 equivalents of Hünig's base. In situ addition of three equivalents of $\mathrm{HBF}_{4}$, followed by precipitation provides mono-protonated $\mathbf{5}$, with protonation occurring primarily on the more electron rich benzimidazole (see below). Adding additional $\mathrm{HBF}_{4}$ can drive this through to 7 . The di-protonated species (6) could not be cleanly isolated without contamination from either the mono or tri-protonated species. Dimers 4, $\mathbf{5}$ and 7 exhibited distinct optical and spectroscopic behaviour (Figure 2). The differences in the ${ }^{1} \mathrm{H}$ NMR spectra of these compounds are consistent with the expected changes in electron density (Figure 3, Table S1).

Although we are not able to conclusively assign the state of protonation by mass spectrometry, the ESI-MS spectra of both 5, and 7, support the proposed structures, with molecular ions

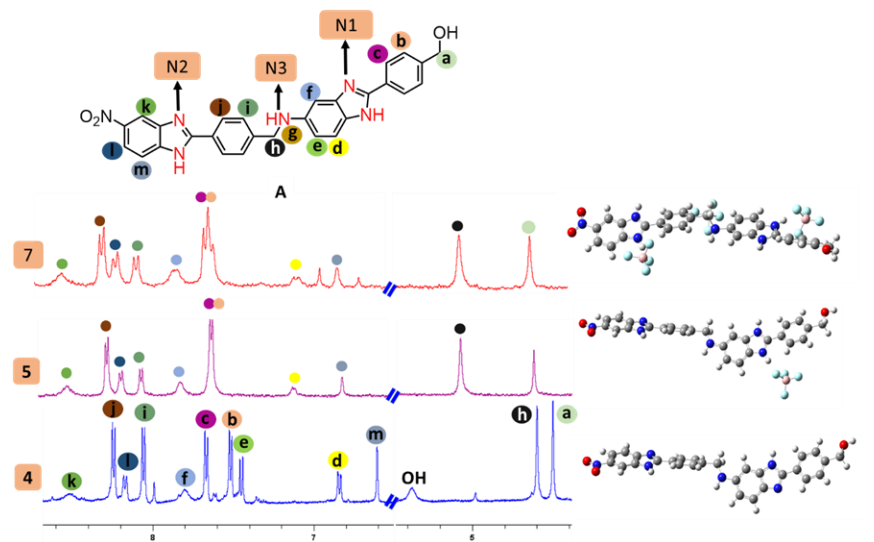

Figure 3. Partial ${ }^{1} \mathrm{H}$ NMR of neutral $\mathbf{4}$, monoprotonated $\mathbf{5}$ and fully protonated $\mathbf{7}$. 


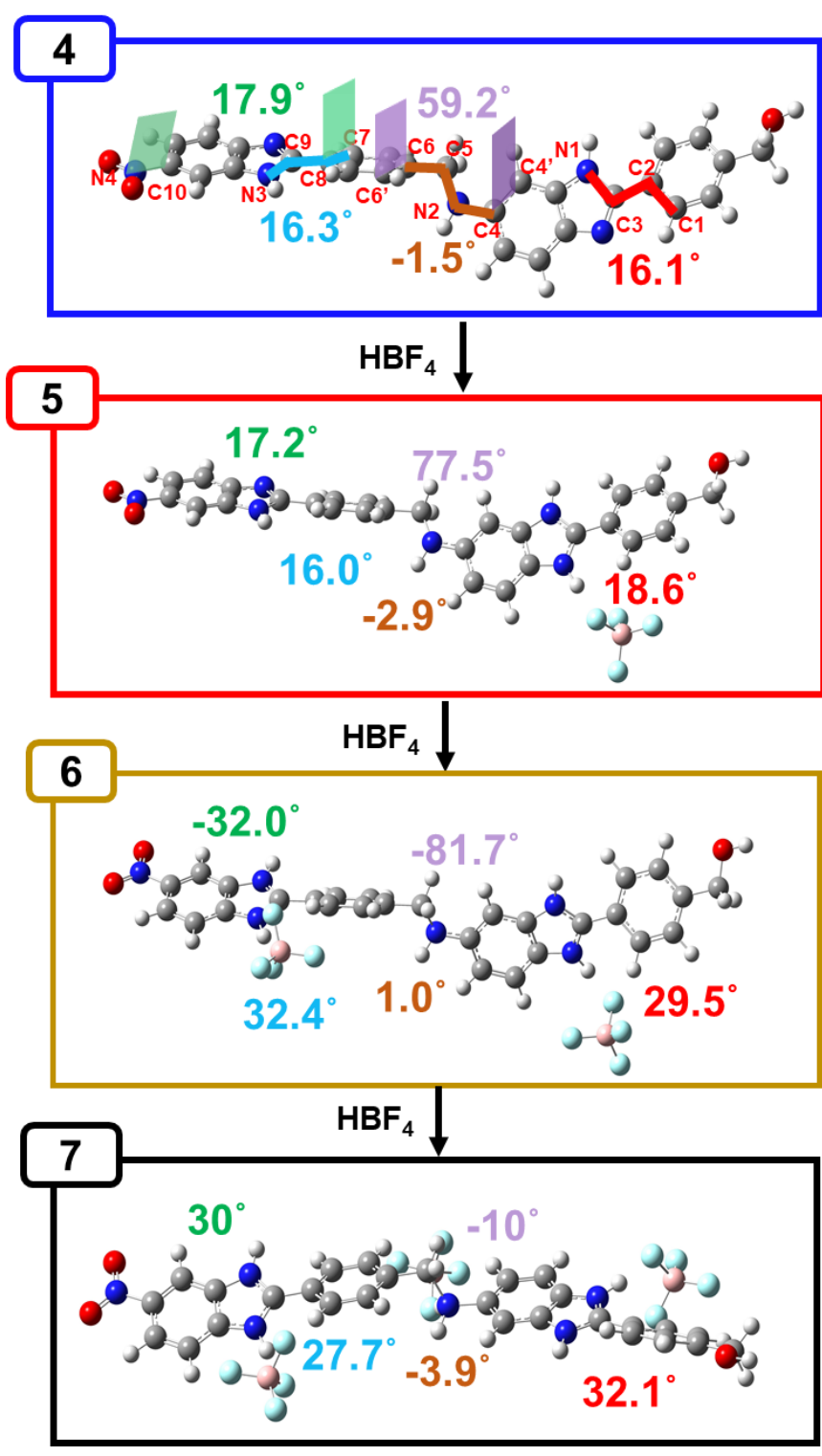

Figure 4. The $\omega B 97 X D / 6-311 G(d, p)$ calculated minimum energy structures of neutral and protonated dimeric skeletons.

consistent with both mono and di-protonated species $(\mathrm{m} / \mathrm{z} 491$ \& 246 respectively). For compound 7 , an $M+1$ ion at $\mathrm{m} / \mathrm{z} 742$ and +2 at 371 were also observed, consistent with what we believe could be a trimer, likely a figment formed during mass spectrometry. Notably, these ions are also observed in $\mathbf{5}$, but the spectral patterns are different, suggesting differential fragmentation depending on protonation state. Spectra obtained in negative mode are consistent with $\mathrm{BF}_{4}-$ present in both 5 and 7 (and not in 4), supporting the identity of the counterion. These spectra are reproducible from separate synthetic batches.

To obtain a more detailed understanding of these materials and the protonation process, DFT calculations at $\omega B 97 X D / 6$ $311 \mathrm{G}(\mathrm{d}, \mathrm{p})$ level of theory were conducted. The minimum energy structures of 4, 5, and 7, were determined (Figure 4, Figure S1).

Comparing the energetics of each possible mono-, di-, and tri-protonated skeleton in both gas phase and DMSO (IEFPCM solvation model), and either in the presence or absence of counterions, strongly suggested that the most probable species at each protonation stage was consistent with intuition (Figure
4; see Figure S1 for the other, higher energy, protonated monodi and tri-protonated skeletons). The electron-rich benzimidazole's N1 is the most basic site, followed by N3 on the nitro substituted benzimidazole, with the aniline N2 being the third most basic site. Protonation, however, significantly distorts the conformation of these systems (Figure 4). The benzimidazolium and phenyl rings are 16.10 from coplanar in 4, maintaining conjugation. However, protonation bends the electron-rich system out of alignment to 18.6 o in $\mathbf{5}$, and a predicted 32.10 in the fully protonated 7; breaking conjugation. This would be expected to impact donor-acceptor charge transfer, likely affecting the resulting optical properties. We see similar deviations from the ideal for the electron poor benzimidazole, and even in the geometry of the nitro group with the benzimidazole. Surprisingly, we do not predict any dissociation of the counterions from the bMeBAB in DMSO even for 7 , despite this type of analysis generally being the focus of our work. ${ }^{[20]}$ These hypotheses are supported by experimental work: one of the possibilities is that dilution of these systems in DMSO changes the protonation states; however, addition of excess acid does not materially change the NMR of dissolved 7 while it does convert dissolved 4 to match $\mathbf{7}$, suggesting that 7 does rest as the trication (Figure S2).

These distortions are expected to affect the optical behaviour of these molecules due to their impact on the key molecular orbital (MO) and energies. Both the MOs and timedependent density functional theory (TD-DFT) calculated parameters were estimated using $B 3 L Y P / 6-311+G(2 d, p)$, in DMSO. The key calculated frontier orbitals, LUMO+2, LUMO+1, LUMO, HOMO, and HOMO-1, HOMO-2, are shown in Figure 5. Additional MOs are provided in Figure $\mathbf{3 3}$ and Table S2. The most likely transitions, and consequently the MOs involved, were selected by balancing maximizing oscillator strength ( $f$ ) and minimizing excitation energies ( $E_{\text {excitation }}$ ). These calculations provided the expected $\lambda_{\mathrm{abs}}$ max.

As shown in Figure $\mathbf{5}$ and Figure S3, in $\mathbf{4}$, the energies of the key MO assigned to be HOMO-2 $(-6.60 \mathrm{eV})$ and LUMO $(-2.87 \mathrm{eV})$ of the benzimidazole moiety are only slightly lower than those of 5 (HOMO -5.89 , and LUMO+1; -2.14 eV). Mono or diprotonation of the dimer only slightly increases its key $\mathrm{MO}$ energy levels compared with the neutral form suggesting that the degree of electron transfer between the donor and acceptor fluorophores should be broadly similar, although differences in aggregation might result in experimental differences. Fully protonated 7 behaves very differently. Its HOMO-1 $(-9.38 \mathrm{eV})$, is far lower than that of 4 , while its LUMO orbital (LUMO $=-1.28$ $\mathrm{eV}$ ) is much higher. This large gap in donor-acceptor orbital energy both reduces the ability of electrons to transfer from the donor unit to the acceptor, and also perturbs local excitation. This should quench fluorescence.

Protonation is known to decrease conjugation in arylbenzimidazole systems, altering electron accepting ability and inducing changes in colour and optical behaviour. ${ }^{[21]}$ The significantly differential optical behaviour of $\mathbf{4 , 5}$ and $\mathbf{7}$ was the phenomenon that initially elicited our interest in this current study, solutions of the former fluoresce under sunlight while those of the latter two are more muted. To quantify this result, we measured their protonation-dependent optical behaviour at 


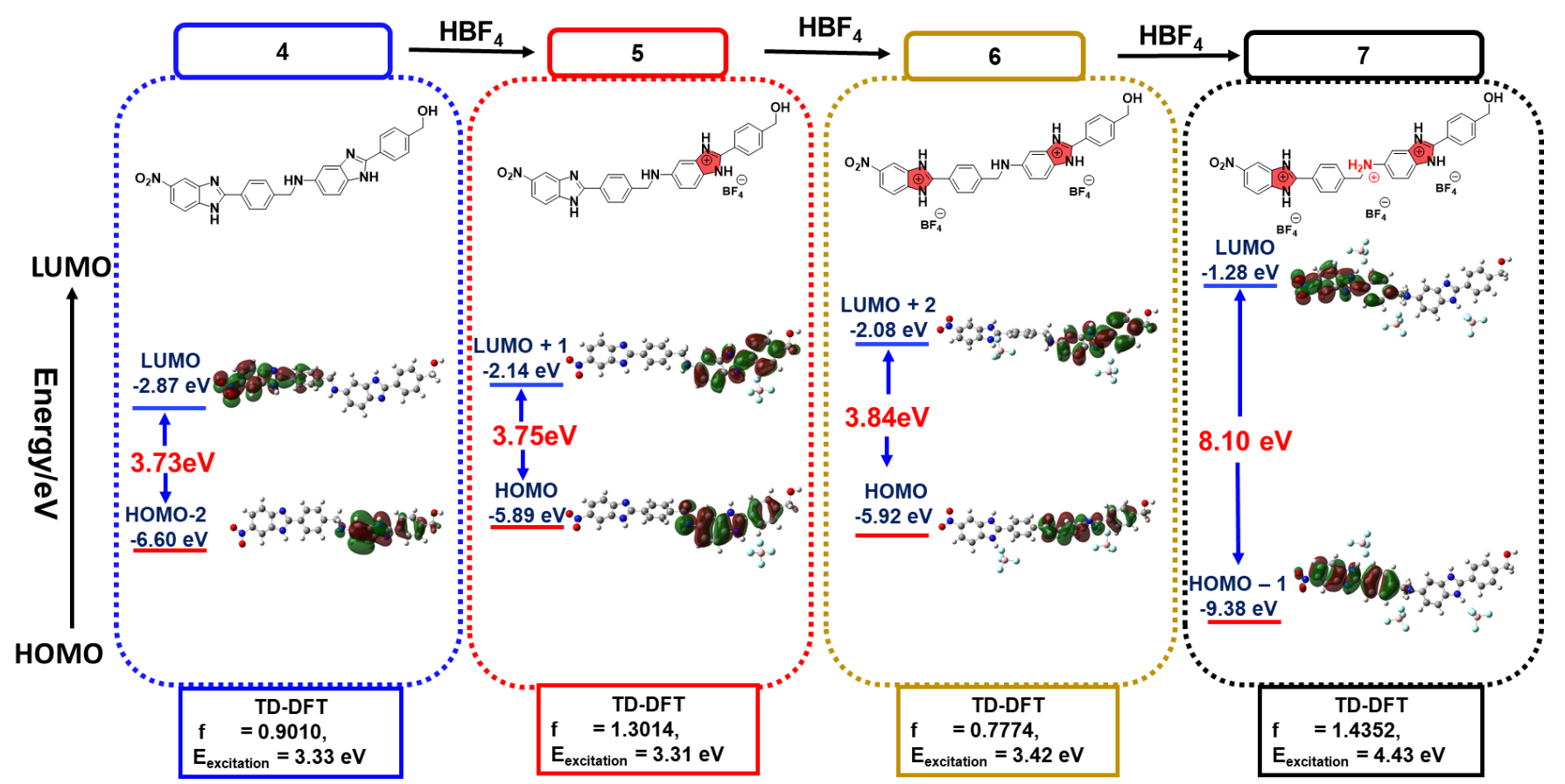

Figure 5. The DFT predicted molecular orbitals, band gap, and the TD-DFT calculated optical parameters of neutral and different protonated skeletons.

$0.03 \mathrm{mM}$ in DMSO-d 6 (Figure 2). Each molecule has different optical properties. All demonstrate long-wavelength bands at around $302 \mathrm{~nm}$ (the principal peak in the UV-Vis spectrum) albeit with different intensities. The two lower-energy absorption bands around 360 and $425 \mathrm{~nm}$ are lower intensity, except in $\mathbf{4}$ where all three are similar. We propose that the 302 $\mathrm{nm}$ peak likely arises from the free molecules as it is consistent with the calculated $\lambda_{a b s, m a x}$, while the two peaks at longer wavelengths ( $360 \mathrm{~nm}$ and $425 \mathrm{~nm}$ ) likely represent the interference from hydrogen-bonded dimer aggregation, or are due to other $\pi-\pi^{*}, \mathrm{n}-\pi^{*}$ transitions that are easier to access in the aggregates. A complete emission spectrum was obtained by excitation at two different wavelengths, 300 and $450 \mathrm{~nm}$. Others have reported the emergence of similar new red-shifted absorption bands as a function of aggregation for both benzimidazole skeletons and other dyes. ${ }^{[22]}$

As shown in Figure 2a, upon protonation of neutral dimer 4, the absorption intensity increases significantly, accompanied by a moderate change in the $\lambda_{\mathrm{abs} \text {, max. }}$. The absorption and emission spectra of neutral skeleton $\mathbf{4}$ are like those of monoprotonated 5; however, 5 exhibits a stronger UV-Vis intensity than 4, and

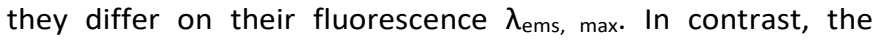
fluorescence $\lambda_{\text {ems, max }}$ blue-shifts from $427 \mathrm{~nm}$ to $399 \mathrm{~nm}$ and $352 \mathrm{~nm}$ upon protonation to $\mathbf{5}$ and $\mathbf{7}$, respectively (Figure 2c,d). Furthermore, when excited at the longer wavelength, fluorescence of fully-protonated $\mathbf{7}$ is effectively quenched, precisely as predicted by the computational calculations (Figure 2d), and as would not be expected for any of the other protonation states, further supporting the characterization and supporting the solution-phase existence of this tri-cation. The dual fluorescence behaviour in these skeletons can be ascribed to a combination of local excitation of the electrons from the benzimidazole $\pi$ to the benzimidazole $\pi^{*}$, and variations in the intramolecular charge transfer (ICT) due to changes in the relative conformation, geometry and distance between the benzimidazoles. The highly conjugated nature of bMeBABs makes ICT the likely dominant mode of fluorescence; this would be highly sensitive to conformational effects that break conjugation. We propose that the presence of photo-induced electron transfer between the benzimidazolium units coupled with a reduced degree of $\pi$-conjugation between the phenyl and benzimidazolium moieties, as they contort out of planarity upon protonation, is responsible for the observed optical behavior. This is exacerbated by the change in the angle of the nitro group on the electron-poor system as it also distorts out of conjugation as protonation increases. This effect arises from both changes in electron density and steric pressure from the counterions trying to mask the charge. Regardless, the decrease in conjugation across all moieties of the molecule in $\mathbf{7}$ will decrease local excitation and charge transfer and should lead to fluorescence quenching. In contrast, for both neutral $\mathbf{4}$ and monoprotonated 5 (and putative $\mathbf{6}$ ), electrons can transfer from the donor phenyl-benzimidazole to the acceptor system (Figure S3, Table S2). A curious effect is observed upon the second protonation to putative 6 where the acceptor system inverts so that the nitro group now sits on the back of the molecule, subtly altering the electronic structure of the ground and excited states. These predictions are reflected in the fluorescent spectrum as breaking conjugation should reduce absorption at higher wave numbers. This is what is observed.

The calculated key orbital energy differences $(\Delta E)$ for $\mathbf{4}$ and 5 are 3.73 , and $3.75, \mathrm{eV}$, respectively, while that for fully protonated $\mathbf{7}$ is an insurmountable $8.10 \mathrm{eV}$. The predicted trend in the orbital gap for these protonated species agrees well with the experimental observations: $\mathbf{7}$ quenches fluorescence at the higher wavelengths. Similarly, the computationally predicted $E_{\text {gap }}$ of $\mathbf{4}$ and $\mathbf{5}$ are lower than for $\mathbf{7}$ which shows a potent blueshift and have a very high frontier orbital energy gap. This again 
agrees with the observed behaviour, supporting the contention that this is the species in solution.

A precise match between the calculated $\lambda_{\mathrm{abs}, \max }$ and $\lambda_{\mathrm{ems} \text {, max }}$ and those observed by experiment was not expected as these molecules clearly exist as a mixture of isolated and aggregated forms in solution and our calculations necessarily ignore the emergent intra- and inter-molecular interactions that would influence these values. However, we can compare relative trends. The calculated oscillator strength (f) of the assigned main orbital transitions is proportional to the quantum yield, higher values indicate a greater probability of photon emission/absorption during an electron transition. Neutral dimer 4, with the weakest experimental absorption intensity, has the smallest $f$ value $(0.90)$; mono-protonated $\mathbf{5}$, with an intermediate absorption intensity, has an intermediate $f$ value of 1.30; while fully-protonated 7 , with the strongest signal intensity has the highest oscillator strength at 1.43. The differential optical behaviour as a function of protonation, shows this system's sensitivity to its environment. This optical behaviour is of more than academic interest, as if it is further affected by the formation of pseudorotaxanes, it can be used as a simple chromatic sensor for supramolecular complex formation; a far simpler read-out than relying on complex intersectional multi-instrumental analytical techniques. This in situ characterization would make these even more privileged structures. To explore this possibility we first investigated the aggregation and solvent-dependent behaviour.

Photophysical response varies as a function of solvent and concentration/aggregation As we seek to use these for selfassembly applications, and as aggregation appears to be a challenge at even low concentrations regardless of protonation state, we needed to better understand the relationship between aggregation state, solvent choice, and analyte concentration in these systems. As the tri-protonated species would best be able to chelate to cation-recognizing macrocyclic units, and hence the most useful for any application; this species, 7, which alone of the examined species was effectively non-fluorescent at $0.03 \mathrm{mM}$ in DMSO when irradiated at 450 $\mathrm{nm}$, was used for all further studies.

The UV-Vis absorption spectra (300-450 nm) of the fully protonated axle 7, were collected over a large concentration range, and in several solvent systems. The low energy $\lambda_{a b s, \max }$ peak of $\mathbf{7}$ exhibits an extreme concentration-dependent redshift (Figure 6a) to higher wavenumbers as the concentration increases. This peak is not present in samples with concentrations of $0.1 \mathrm{mM}$ or below. Decreasing the concentration of axle from $3 \mathrm{mM}$ to $0.01 \mathrm{mM}$ in DMSO, leads to a significant change in the color from a deep yellow to colourless (Figure $6 \mathbf{b}$ ). The colour gets fainter and fainter and then disappears at $0.1 \mathrm{mM}$, the same "cut-off" value as noted in the UV-vis data. The bMeBAB also exhibits significant linear fluorescence enhancement upon increasing of the concentration; overcoming the fluorescence quenching observed at $0.03 \mathrm{mM}$ concentration (see above, Figure $6 \mathrm{c}$ ) with a distinct blue-green emission centred at $528 \mathrm{~nm}$ (Figure 6b). A concentration-dependent decrease is clearly observed down to 0.3 , but it drops off markedly below this point. This discontinuity
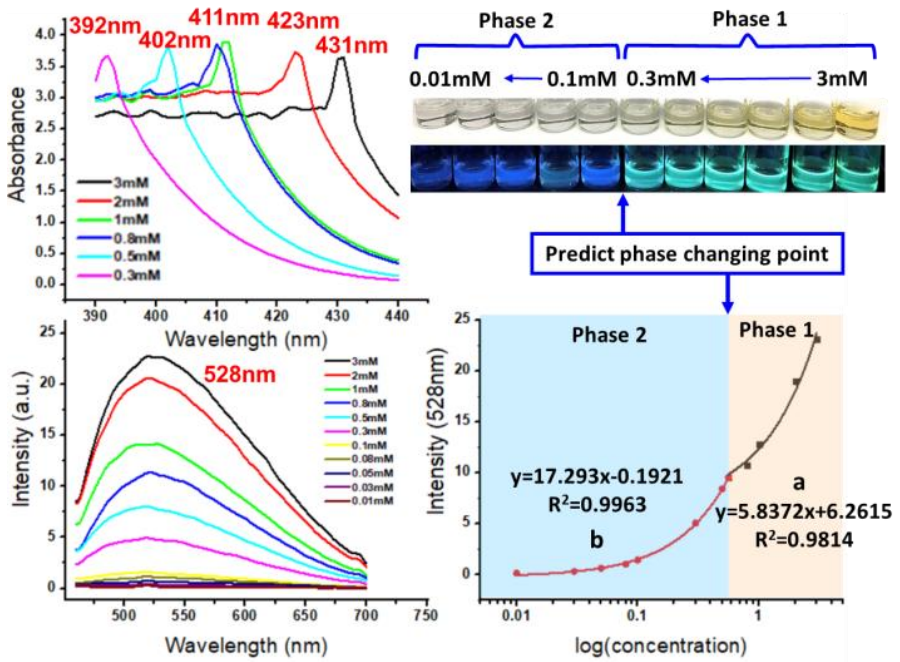

Figure 6. (a)The UV-Vis spectra of $3 \mathrm{mM}-0.3 \mathrm{mM} 7$ in undried DMSO; (b) photographs of the samples of $\mathbf{7}$ used to generate Figure 5 under ambient light (top) and under excitation at $365 \mathrm{~nm}$ (bottom) in undried DMSO; (c) the fluorescence spectra of $\mathbf{7}$ in undried DMSO (3 mM-0.01 mM) with excitation at $450 \mathrm{~nm}$; (d) Fluorescence intensity plotted against concentration with the equations of the two curves. (Note the discontinuity at $0.3 \mathrm{mM}$ )

in behaviour is clearer when the data is plotted (Figure 6d), showing that we have two different mechanisms of fluorescence in play. We believe that this may be attributed to aggregation induced emission behaviour.

Absorption/emission shifts like that in Figure 6a are known but are very rare and we are unaware of any system with such a drastic change over such a small concentration change. ${ }^{[23]}$ There is little change in the amplitude of the signal. Consequently the shift likely arises from a combination of the emergence of a new species, that replaces the computationally calculated energy minimum (Figure S1, S3); the emergence of additional non-covalent, ion-pairing and $\mathrm{H}$-bonding interactions between functional groups on adjacent axles; the restriction of molecular rotation and vibration due to aggregation; and/or the interference of intramolecular charge transfer by intermolecular processes due to the increase in concentration. To try to narrow down the contributing causes we attempted to disrupt the aggregates by changing the solvent, believing that the trace water in the DMSO could be affecting molecular solvation, as reported for similar systems. [24]

Employing DMSO $\left(d_{6}\right)$, pre-dried over previously washed $4 \AA$ molecular sieves, induced no change in the fluorescent behaviour, but did induce a red-shift compared to the slightly wet sample (Figure S4; Table S3). Considering that water could be expected to break-up aggregates, and that aggregates are likely responsible for the extreme shift in the $\lambda_{\mathrm{abs} \text {, max, }}$ one would anticipate that a solution in wet DMSO would behave like a lower concentration sample in DMSO in terms of aggregation(Figure S4; Table S3).

We investigated the concentration-dependent optical behaviour of 7 in both wet DMSO and DMF (10\% v/v water in each). By inspection, adding water makes a big difference (Figure 6 vs Figure 7 and Figure S4), likely affecting aggregation and/or conformational distribution and consequently, its optical properties. Water likely better solvates these polycations, disrupting aggregation. 

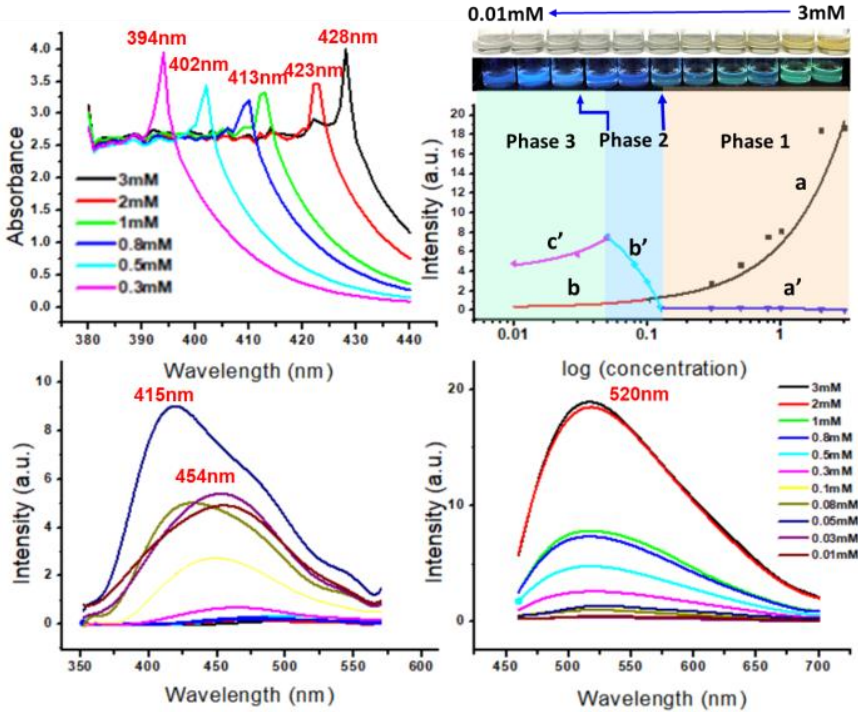

Figure 7. (a)The UV-Vis spectra of $7(3 \mathrm{mM}-0.3 \mathrm{mM})$ in 9:1-DMSO: $\mathrm{H}_{2} \mathrm{O}$; (b) The fluorescence spectra, excited at $300 \mathrm{~nm}$ of 7 ( $3 \mathrm{mM}-0.01 \mathrm{mM})$ in 9:1-DMSO: $\mathrm{H}_{2} \mathrm{O}$; (c) ambient light (top), excitation at $\lambda=365 \mathrm{~nm}$ (bottom) appearance of 7 in 9:1DMSO: $\mathrm{H}_{2} \mathrm{O}$; (d) The fluorescence intensity of various concentrations at $520 \mathrm{~nm}$ emission (black, red) and $415 \mathrm{~nm}$ emission (blue, cyan, pink); (e) The fluorescence spectra, excited at $450 \mathrm{~nm}$, of 7 ( $3 \mathrm{mM}-0.01 \mathrm{mM}$ ) in 9:1-DMSO: $\mathrm{H}_{2} \mathrm{O}$

The presence of $10 \%$ water within the solution, keeps the solutions colorless at higher concentrations than does pure DMSO (Figure 7c, Figure S4c vs Figure 6c); this suggests that the yellow tint may arise from aggregates rather than the monomer alone. Increasing the concentration of 7 in the $10 \%$ water in DMSO solution from $0.3 \mathrm{mM}$ to $3 \mathrm{mM}$ leads to a $U$-shaped curve in amplitude and the same definite red shift in the $\lambda_{\mathrm{abs}}$ max as was seen in dry DMSO; curiously the peaks are sharper in this spectrum than they were for pure DMSO where they are more Gaussian. Again, like before, this peak is not present at concentrations $0.1 \mathrm{mM}$ or below. The fluorescent behaviour is more complicated in this mixed solvent system. As the concentration of 7 rises from 0.01 to $0.05 \mathrm{mM}$, the fluorescence emission at $400-450 \mathrm{~nm}$ (excitation at $300 \mathrm{~nm}$ ) gradually increases (Figure $7 \mathbf{b}$ ), with a significant shift in the $\lambda_{\text {ems, max. As }}$ concentration continues to rise, amplitude starts to fall to 0 , and fluorescence is effectively fully quenched at concentrations above $0.5 \mathrm{mM}$. Conversely, when excited at $450 \mathrm{~nm}$, we see a steady increase in emission at $520 \mathrm{~nm}$, effectively reaching maximum signal at $3 \mathrm{mM}$ (Figure 7e). This differential behaviour suggests that an isolated molecule, or more likely a dimer, emits at $415 \mathrm{~nm}$, but that this band is quenched as aggregates start to form as the concentration increases. The concentration of monomer above the critical aggregation concentration would remain constant and we would not expect to see a decrease in emission, but rather a stabilization. It is likelier that the $415 \mathrm{~nm}$ peak is due to a lower ordered aggregated form or different types of aggregation that effectively ceases to exist as the concentration continues to rise, producing species that emit at $520 \mathrm{~nm}$.

Therefore, 7 experiences a concentration dependent, twophase (Phase 1 is between $3 \mathrm{mM}$ to $0.3 \mathrm{mM}$ and phase 2 is 0.1 $\mathrm{mM}$ to $0.01 \mathrm{mM}$ ), aggregation process with each one having its own relationship between concentration and emission maximum (Figure 7d). Changes in absorption at the lower concentrations (0.01 to $0.5 \mathrm{mM})$ are extremely sensitive to small changes demonstrating the potential of fully protonated 7 to act as a chemical sensor. Interpolation suggests the mechanism changes at $0.56 \mathrm{mM}$, however there is clearly a transition zone between 0.5 and $0.3 \mathrm{mM}$ where we have a mixture of the mechanisms coexisting. [25]

We considered that the effect could be due to changes in solvent viscosity, so to discount this possibility we examined 7 in a 9:1-DMF: $\mathrm{H}_{2} \mathrm{O}$ system (Figure S5). Consistent with the DMSO system, increasing the concentration of $\mathbf{7}$ enhanced the intensity and appearance of sharp absorptions in the UV-vis spectrum as in Figure 6a and 7a. This is not solvent specific behaviour; it appears to be a feature of the molecule.

To provide supportive evidence of this aggregation behaviour, we employed dynamic light scattering (DLS) (Figure 8). The DLS data shows that aggregates are present, and that the size varies based on solvent, but is little affected by concentration above $0.08 \mathrm{mM}$; below $0.08 \mathrm{mM}$ particles are not observed by DLS, suggesting this might be close to the critical aggregation concentration (CAC). This is very close to the $0.1 \mathrm{M}$ cut-off we observed in the optical data, strongly supporting our contention that the unusual photophysical behaviour arises from aggregation. The lines in the DLS are essentially flat within the error of the measurements, indicating that a single species seems to dominate the aggregation form regardless of the concentration once the CAC is attained (Figure 8). Consistent with the above optical observations, using mixed solvent systems changes the particles that are present: they are far smaller (100 nm vs $600 \mathrm{~nm}$ at $3 \mathrm{mM}$ concentration) when water is introduced, again supporting our hypothesis that water disrupts the aggregates by better solubilizing the tri-cations. But again, an aggregate is observed across this concentration range, with nothing detectable by DLS below $0.08 \mathrm{mM}$ suggesting that this might be close to the CAC for this compound in both solvents, again inline with the optical response. This concentration of bMeBAB is far lower than will be employed for the supramolecular assembly chemistry we intend to use these systems for, suggesting that aggregation might be a serious concern. Polymers, rather than these simple model dimers, will

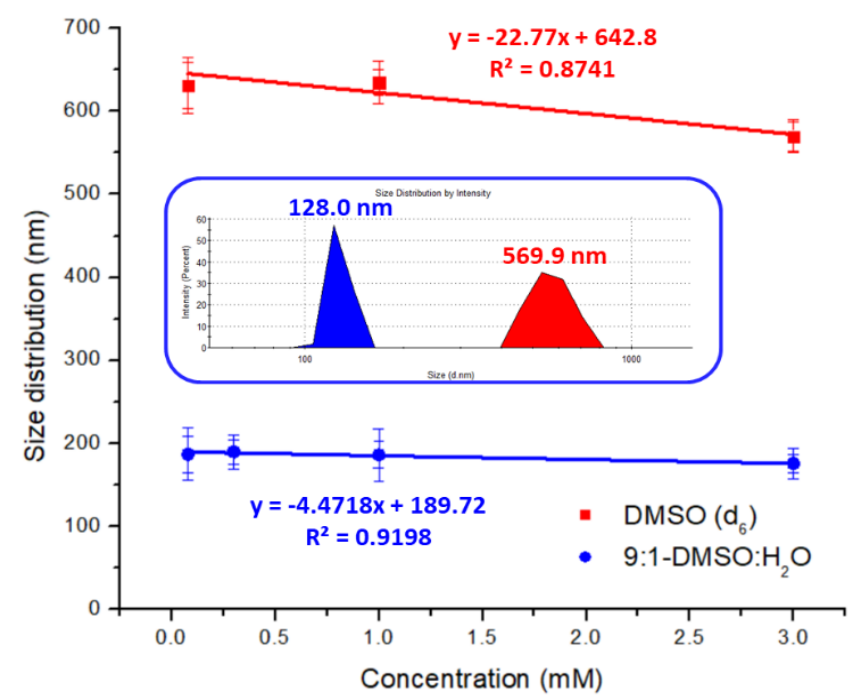

Figure 8. Linear trend between concentration of $\mathbf{7}$ and solvodynamic radius in the different solvent systems. In neither system were particles observed below $0.1 \mathrm{mM}$. Data must be interpreted carefully as DLS can be unreliable for fluorescent samples. 


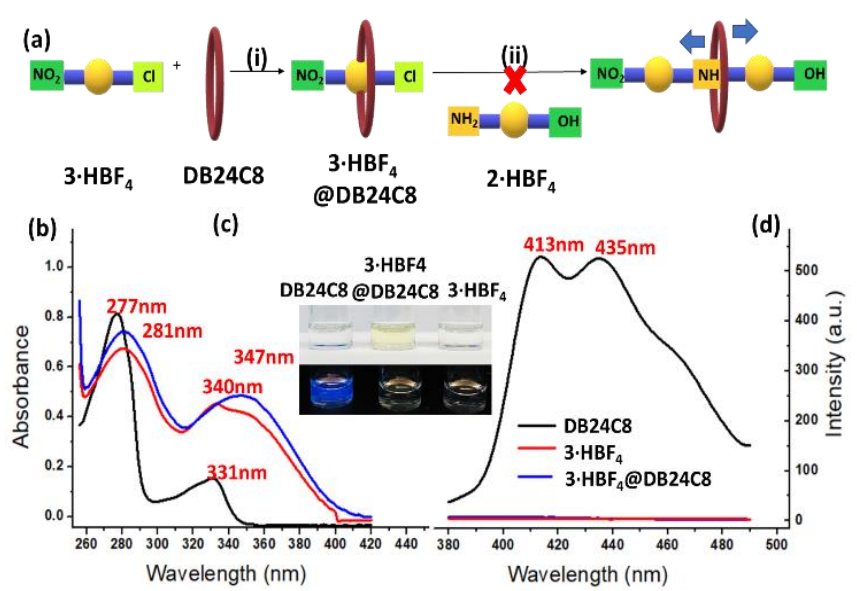

Figure 9. Pseudorotaxane formation. (a) synthesis route toward 3.HBF $@$ DB24C8, (i) $\mathrm{CHCl}_{3}, 25^{\circ} \mathrm{C}$, (ii) 0.2 equiv. Nal, $25^{\circ} \mathrm{C}$. (b, d) UV-vis-Fluorescence spectra of host, guest and [2] pseudorotaxane in DMSO; (c) ambient light, excitation at $\lambda=365 \mathrm{~nm}$ appearance of host, guest and [2]pseudorotaxane in pure dry DMSO $\left(d_{6}\right)$. The measurement of UVVis-fluorescence was carried out with $0.3 \mathrm{mM}$ solution of isolated host, guest and [2] pseudorotaxane $\left(3 \cdot \mathrm{HBF}_{4} @ \mathrm{DB24C8}\right)$ in $\mathrm{DMSO}\left(\mathrm{d}_{6}\right)$.

be used at a lower molarity (although a higher mass concentration) and are likely to behave differently and will need to be investigated separately. Regardless, these results suggest that our planned chemical functionalization of the aryl rings to increase solubility and tune macrocycle affinity, will likely be necessary.

Both the optical and sizing data strongly suggests that a single class of aggregate is formed at higher concentrations, and that this aggregate begins to emerge above $0.08 \mathrm{mM}$. Addition of water weakens the interactions resulting in smaller aggregates, but it does not change the CAC significantly. These aggregates show remarkable concentration-dependent redshifting making them extremely curious structures deserving of further study.

Protonated bis-benzimidazoliums as chromophoric axles and crown ethers: Formation of optically detectable pseudorotaxanes

Poly(bMeBABs) are promising axles for the generation of poly(pseudorotaxanes) with complementary macrocycles such as crown ethers. We begin this investigation using a combination of ${ }^{1} \mathrm{H}$ NMR, high resolution mass spectrometry (HRMS), UV-Vis and fluorescence spectroscopy as the fluorescent behavior of the bMeBABs makes them their own sensors. We began our investigations with the most soluble monomeric precursor of 3, its $\mathrm{HPF}_{6}$ salt, $\mathbf{3} \bullet \mathrm{HPF}_{6}$ (Scheme S2, Figure S6 and S7, and Table S4), but switched to using $3 \bullet \mathrm{HBF}_{4}$ as we found the chemistry more reproducible, and recommend the same to anyone looking to investigate these systems. After screening several crown ethers (Figure S8-S10), dibenzo-24crown-8 (DB24C8) was both computationally predicted, and experimentally demonstrated, to be a good match size-wise (21 atoms was a bit small, 27 a bit large). The optimized geometries of the various potential host-guest complexes obtained from tuning either the counterion (Figure S11) or the host (Figure

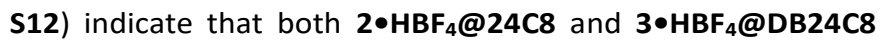
provide the most favorable host-guest interaction energy and optimal solvation free energy. The latter's inherent fluorescence makes it more useful for the current study.
Thus, we accessed [2]pseudorotaxane, 3•HBF $4 @ D B 24 C 8$ by combining the two components in $\mathrm{CH}_{3} \mathrm{Cl}$ at room temperature. The complex was then treated with $\mathbf{2} \cdot \mathbf{H B F}_{4}$ to attempt to make the [2] pseudorotaxane of bMeBAB 7 (Figure 9, Figure S13). However, after purification, only uncoordinated dimer 7 and a small amount of residual 3• $\mathbf{H B F}_{4} @ \mathbf{D B 2 4 C 8}$ were isolated. The latter is distinguishable from the uncoordinated $\mathbf{3}$ due to moderate changes in the ${ }^{1} \mathrm{H}$ NMR chemical shifts of the aryl protons as a result of $\mathrm{NH} \cdots \mathrm{O}$ hydrogen bonding, $\mathrm{CH} \cdots \mathrm{O}$ and $\pi-$ stacking interactions between the electron poor benzimidazolium and phenyl rings of the axles and the electron rich rings of DB24C8 (Figure S13b; Table S5). This is suggestive of a complex, but the data could arise from either exo (face-toface interaction) or endo (inclusion) complexation. The former is likely to be more transient than the latter although both are in equilibrium.

However, the DOSY NMR, optical, and mass spectrometric analyses all suggest that $\mathbf{3} \cdot \mathbf{H B F}_{4} @ \mathbf{D B 2 4 C 8}$ is a strongly associated endo complex (Figure 9, Figure S13). DOSY visualizes diffusion; DB24C8 and $\mathbf{3} \bullet \mathrm{HBF}_{4}$ have different solvodynamic radii and different rates of diffusion. ${ }^{[26]}$ However, if they assemble, they would show the same, slower diffusion, and this is what is observed (Figure S13c). Similarly, $\mathbf{3} \cdot \mathbf{H B F}_{\mathbf{4}}$ has two absorbance maxima in the UV-vis spectrum at $281 \mathrm{~nm}$ and 340 $\mathrm{nm}$, while DB24C8 absorbs at $277 \mathrm{~nm}$ and $331 \mathrm{~nm}$ (Figure 9b). When the two are mixed together, the axle dominates the spectrum, but there is no evidence of the DB24C8 peak at 331 $\mathrm{nm}$; instead, there is a slight red-shift to $347 \mathrm{~nm}$ with a concomitant increase in signal amplitude. This is also seen at the lower wave-number signal where the intense DB24C8 absorbance at $277 \mathrm{~nm}$ completely disappears, and the mixture exhibits a stronger signal at $281 \mathrm{~nm}$ resembling the free axle. DB24C8 is highly fluorescent (Figure 9d), with emission at 413 $\mathrm{nm}$ and $435 \mathrm{~nm}$ (when excited at $370 \mathrm{~nm}$ ); however, these two emission peaks are completely quenched in the mixture. Finally, this effect can be seen with the naked eye (Figure 9c). In sunlight, both isolated $\mathbf{D B 2 4 C 8}$ and $\mathbf{3} \cdot \mathbf{H B F}$ are colorless; however, the $1: 1$ mixture is yellow $\left(0.3 \mathrm{mM}\right.$, DMSO $\left(d_{6}\right)$, room temperature). When excited at $\lambda=365 \mathrm{~nm}, \mathbf{D B 2 4 C 8}$ is highly fluorescent, but neither $\mathbf{3} \bullet \mathbf{H B F}_{4}$ nor the mixture of the two molecules are; the interaction is good enough that the axle quenches the crown ether's fluorescence. DB24C8 does not exist as an optically-active independent species when in a 1:1

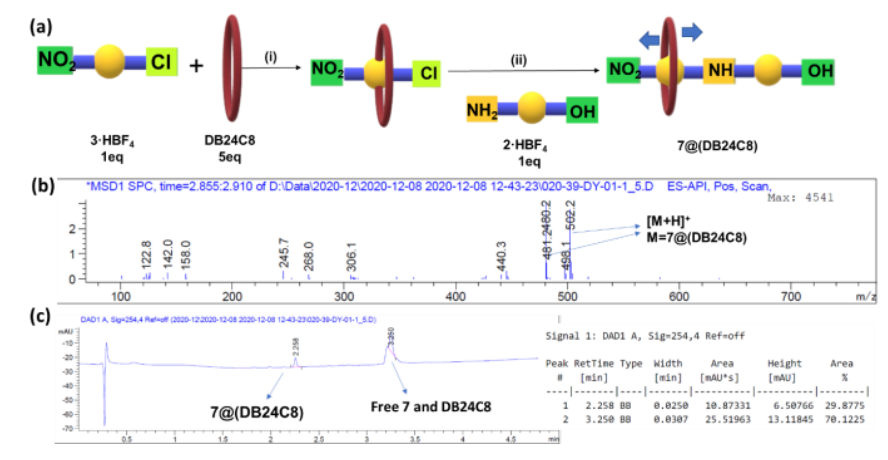

Figure 10. (a) Synthetic route toward 7@DB24C8, (i) $\mathrm{CH}_{3} \mathrm{CN} / \mathrm{H}_{2} \mathrm{O}, 25^{\circ} \mathrm{C}$ (ii) 0.2 equiv. $\mathrm{Nal}, 50^{\circ} \mathrm{C}$ (b) MS(+)spectra of 7@DB24C8. (c) LC spectra of 7@DB24C8. ratio with $3 \bullet \mathrm{HBF}_{4}$ in DMSO. 
Tightly-bound pseudorotaxanes sometimes survive the ene rgies used in mass spectrometry; this is the case here where the electrospray spectrum provides an exact match for the complex at $\mathrm{m} / \mathrm{z}$ 738.2631, with an isotope pattern consistent with 3•HBF 4 @DB24C8. This assembly would be unlikely to be observed for a simple exo (non-inclusion) complex. The existence of the [2]pseudorotaxane of these bMeBABs as a kinetically stable complex is supported by its detection through LC-MS analysis (Figure 10, b-c ); injection of the crude reaction mixture provides two peaks with ratios consistent with the NMR data for complexed and uncomplexed axle-crown ether with the second peak providing a mass of 480.2 and 502.2, highlighting the formation of the pseudorotaxane 7@DB24C8 (proton and sodium complex respectively). The second peak, with a clear shoulder, in the spectrum shows masses for both the crown ether and the axle separately. If this was in fast equilibrium on the column, the signal would be smeared along the baseline as two broad peaks; that it is not suggests that a kinetically stable complex can be formed. We are currently attempting to acquire a crystal structure of this system to provide more than circumstantial evidence, but the nature of the compound makes crystallization highly challenging. This inclusion complex is of limited independent utility, but its ready formation does tease that higher order assemblies are viable.

These preliminary studies are highly suggestive that pseudorotaxanes can be formed by our novel bMeBABs, which is very promising for the formation of higher order systems on oligomeric and polymeric derivatives. Further investigations into these polymers, their polypseudorotaxane constructs and the determination of the binding affinity of the DB24C8 ethers for these systems are currently underway.

\section{Conclusions}

These bis(methylene-bridged-2-arylbenzimidazoles) bMeBABs are promising colour-changing sensor scaffolds for the generation of pseudorotaxanes, due to their environmentallydependent optical activity. The optical activity of axle alone is highly sensitive to concentration, protonation state, and solvent. As our main purpose is to develop long cross-linked molecular tubes from a pseudorotaxane template, it is essential that all binding sites on the thread are occupied; benzimidazolecrown ether interactions are a promising motif, and the sensitivity of these systems to their environment allows us to monitor protonation, assembly, and undesired aggregation. As mentioned, these systems show the greatest concentrationdependent shift in $\lambda_{\mathrm{abs}, \max }$ and $\lambda_{\mathrm{ems} \text {, max }}$ that we are aware of in the literature for a small molecule making them particularly useful. As students of supramolecular science would understand, this optical activity makes these possibly the simplest scaffolds for determining the presence of a supramolecular complex; an extremely fortunate accident in our chosen system. This will prove highly useful to us in our design of more complex supramolecular constructs, and we believe it will be equally useful to others.

\section{Conflicts of interest}

The authors declare no competing financial interests.

\section{Acknowledgements}

The authors gratefully acknowledge financial support for the project from the Natural Sciences and Engineering Research Council of Canada (JFT: grant \# 2018-06338), the Canadian Tricouncil (JFT: NFRFE-2018-00075), and the American Chemical Society Petroleum Research Fund New Directions Program (JFT: ACS PRF 60765-ND7). SMT and JFT wish to recognize that this work was made possible by the facilities of the Shared Hierarchical Academic Research Computing Network (SHARCNET: www.sharcnet.ca) and Compute/Calcul Canada.

\section{Author Contributions.}

Conceptualization, SMT, JFT; Funding acquisition JFT; Investigation, YX, SMT; Methodology, YX, SMT; Project administration, JFT; Supervision, JFT, SMT; Writing original draft, YX, SMT; Writing - review and editing, All authors.

\section{Notes and references}

[1] a) P. N. Preston, Chem. Rev. 1974, 74, 279-314; b) K. M. H. Nguyen, M. Largeron, Chem. - Eur. J. 2015, 21, 1260612610; c) J. Kulhánek, F. Bureš, O. Pytela, T. Mikysek, J. Ludvík, Chem. Asian J 2011, 6, 1604-1612; d) P. Molina, A. Tárraga, F. Otón, Org. Biomol. Chem. 2012, 10, 1711-1724.

[2] a) Y. Cao, M. Yang, Y. Wang, H. Zhou, J. Zheng, X. Zhang, J. Wu, Y. Tian, Z. Wu, J. Mater. Chem. C 2014, 2, 3686-3694; b) V. S. Padalkar, S. Seki, Chem. Soc. Rev. 2016, 45, 169-202.

[3] a) B. Han, N. Zhou, W. Zhang, Z. Cheng, J. Zhu, X. Zhu, J. Polym. Sci. A: Polym. Chem. 2013, 51, 4459-4466; b) H. Kaur, N. Singh, N. Kaur, D. O. Jang, Sens. Actuators, B 2019, 284, 193-201.

[4] M. Ladinig, W. Leupin, M. Meuwly, M. Respondek, J. Wirz, V. Zoete, Helv. Chem. Acta 2005, 88, 53-67.

[5] E. Horak, P. Kassal, I. Murković Steinberg, Supramol. Chem. 2018, 30, 838-857.

[6] Z. Lu, Y. Liu, S. Lu, Y. Li, X. Liu, Y. Qin, L. Zheng, RSC Adv. 2018, 8, 19701-19706.

[7] J. E. Kwon, S. Y. Park, Adv. Mater. (Weinheim, Ger.) 2011, 23, 3615-3642.

[8] a) G. B. Bodedla, K. R. Justin Thomas, M.-S. Fan, K.-C. Ho, J. Org. Chem. 2016, 81, 640-653; b) G. M. Saltan, H. Dinçalp, M. Kıran, C. Zafer, S. Ç. Erbaş, Mater. Chem. Phys. 2015, 163, 387-393.

[9] E. Horak, R. Vianello, M. Hranjec, S. Krištafor, G. K. Zamola, I. M. Steinberg, Spectrochim. Acta, Part A 2017, 178, 225233.

[10] J. Jayabharathi, V. Thanikachalam, K. Jayamoorthy, Photochem. Photobiol. Sci. 2013, 12, 1761-1773.

[11] M. Marinescu, BMC Chem. 2019, 13, 136.

[12] a) N. Perin, M. Hranjec, G. Pavlović, G. Karminski-Zamola, Dyes Pigments 2011, 91, 79-88; b) T. Chaudhuri, A. Karmakar, S. Ghosh, C. Mukhopadhyay, S. Pal, M. Banerjee, J. Lumin. 2015, 161, 164-173.

[13] a) K. Zhu, V. N. Vukotic, S. J. Loeb, Chem. Asian J. 2016, 11, 3258-3266; b) N. Farahani, K. Zhu, S. J. Loeb, ChemPhysChem 2016, 17, 1875-1880; c) N. Noujeim, K. Zhu, V. N. Vukotic, S. J. Loeb, Org. Lett. 2012, 14, 24842487 ; d) S. Ghosh, A. M. Schmiedekamp, C. Mukhopadhyay, Tetrahedron 2012, 68, 9826-9835; e) D. J. Mercer, S. J. Vella, L. Guertin, N. D. Suhan, J. Tiburcio, V. N. 
Vukotic, J. A. Wisner, S. J. Loeb, Eur. J. Org. Chem. 2011, 2011, 1763-1770.

[14] L. Li, G. J. Clarkson, Org. Lett. 2007, 9, 497-500.

[15] K. Zhu, V. N. Vukotic, N. Noujeim, S. J. Loeb, Chem. Sci. 2012, 3, 3265-3271.

[16] K. Zhu, G. Baggi, S. J. Loeb, Nat. Chem. 2018, 10, 625-630.

[17] J. F. Stoddart, Angew. Chem. Int. Ed. 2017, 56, 1109411125.

[18] P. Shende, B. Prabhakar, A. Patil, Tr. Anal. Chem. 2019, 121, 115687.

[19] a) H.-T. Li, S.-Y. Fan, Z.-P. Li, F.-L. Yu, X.-Q. Hu, J.-C. Cheng, P. Zhang, B.-H. Zhong, W.-G. Shi, Chin. Chem. Lett. 2016, 27, 1630-1634; b) S. J. Loeb, J. Tiburcio, S. J. Vella, J. A. Wisner, Org. Biomol. Chem. 2006, 4, 667-680; c) M. L. Richards, S. C. Lio, A. Sinha, H. Banie, R. J. Thomas, M. Major, M. Tanji, J. C. Sircar, Eur. J. Med. Chem. 2006, 41, 950-969; d) Y. Liu, Y. Lu, M. Prashad, O. Repič, T. J. Blacklock, Adv. Synth. Catal. 2005, 347, 217-219.

[20] a) S. M. Taimoory, K. Twum, M. Dashti, F. Pan, M. Lahtinen, K. Rissanen, R. Puttreddy, J. F. Trant, N. K. Beyeh, J. Org. Chem. 2020, 85, 5884-5894; b) S. M. Taimoory, V. Cataldo, A. Schäfer, J. F. Trant, R. Guterman, Chem. - Eur. J. 2019, Accepted November 2, 2020; c) N. K. Beyeh, I. Diez, S. M. Taimoory, D. Meister, A. L. Feig, J. F. Trant, R. H. A. Ras, K. Rissanen, Chem. Sci. 2018, 9, 1358-1367.

[21] G. Sevinç, B. Küçüköz, H. Yılmaz, G. Şirikçi, H. G. Yaglioglu, M. Hayvalı, A. Elmali, Sens. Actuators, B 2014, 193, 737744.

[22] a) M. Gsänger, D. Bialas, L. Huang, M. Stolte, F. Würthner, Adv. Mater. (Weinheim, Ger.) 2016, 28, 3615-3645; b) K. W. Ding, T.-Q. Li, Z.-X. Ge, J.-H. Bu, Y. Liu, RSC Adv. 2018, 8, 35759-35767.

[23] a) B. L. Cannon, D. L. Kellis, L. K. Patten, P. H. Davis, J. Lee, E. Graugnard, B. Yurke, W. B. Knowlton, J. Phys. Chem. A 2017, 121, 6905-6916; b) N. V. Belko, M. P. Samtsov, G. A. Gusakov, D. S. Tarasau, A. A. Lugovski, E. S. Voropay, J. Appl. Spec. 2019, 85, 997-1005.

[24] I. I. Sahay, P. S. Ghalsasi, ACS Omega 2019, 4, 437-443.

[25] a) Z. A. Tabasi, E. A. Younes, J. C. Walsh, D. W. Thompson, G. J. Bodwell, Y. Zhao, ACS Omega 2018, 3, 16387-16397; b) S. Sahu, Ila, B. Shankar, M. Sathiyendiran, G. Krishnamoorthy, J. Photochem. Photobiol., A 2018, 353, 416-423.

[26] L. Frish, S. E. Matthews, V. Böhmer, Y. Cohen, J. Chem. Soc. Perkin Trans. 2 1999, 669-672. 